Short Communication

\title{
Synthesis and Spectroscopic Studies of New Schiff Bases
}

\author{
Hamid Latif Siddiqui ${ }^{1, *}$ Amjid Iqbal ${ }^{1}$, Saeed Ahmad ${ }^{3}$ and George W. Weaver ${ }^{2, *}$ \\ ${ }^{1}$ Institute of Chemistry, University of the Punjab, Lahore-5400, Pakistan \\ ${ }^{2}$ Department of Chemistry, Loughborough University, Loughborough, LE11 3TU, UK \\ ${ }^{3}$ Pakistan Council of Science and Industrial Research Complex, Lahore, Pakistan \\ * Authors to whom correspondence should be addressed: E-mail: drhamidlatif@yahoo.com; \\ G.W.Weaver@lboro.ac.uk
}

Received: 2 August 2005; revised version received: 10 March 2006 / Accepted: 10 March 2006 / Published: 17 March 2006

\begin{abstract}
Five novel Schiff bases have been prepared from $o$-formylphenoxyacetic acid and a series of aminothiazoles to form a number of potentially biologically active compounds. The structures of these Schiff bases have been characterized using IR and ${ }^{1} \mathrm{H}$ and ${ }^{13} \mathrm{C}-\mathrm{NMR}$ spectroscopy.
\end{abstract}

Keywords: aminothiazoles, $o$-formylphenoxyacetic acid, Schiff bases, biological activity

\section{Introduction}

Schiff bases are used as substrates in the preparation of a number of industrial and biologically active compounds via ring closure, cycloaddition, and replacement reactions [1]. Moreover, Schiff bases are also known to have biological activities such as antimicrobial [2-5], antifungal [4-6], antitumor [7-9], and as herbicides [10]. Schiff bases have also been employed as ligands for complexation of metal ions [11]. On the industrial scale, they have wide range of applications such as dyes and pigments [12]. Keeping in view the facts mentioned, we decided to synthesize new Schiff bases which were predicted to have useful biological activity. The synthesis of other similar Schiff bases, their biological activity, and complex formation are under study. 


\section{Results and Discussion}

The Schiff bases 2a-e were synthesized by condensation of $o$-formylphenoxyacetic acid and aryl aminothiazoles 1a-e by reaction in hot ethanol or dioxane using sodium sulfate as a dehydrating agent. The aminothiazoles were prepared by the known [13] reaction of thiourea with substituted acetophenones in the presence of iodine as oxidant. Good yields were obtained for the Schiff bases, although in some cases the addition of ytterbium triflate was found to improve the yield of product, it acting as a Lewis acid catalyst.

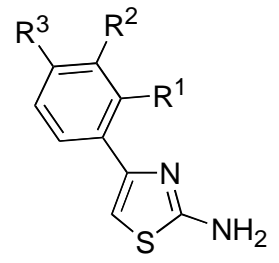

1a-e

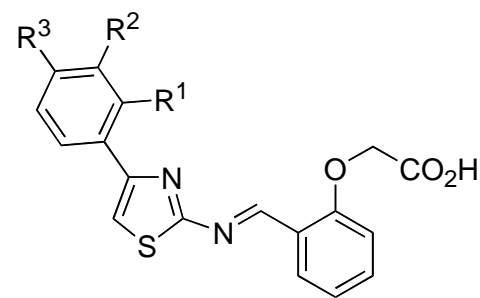

2a-e a $\mathrm{R}^{1}=\mathrm{Br}, \mathrm{R}^{2}=\mathrm{H}, \mathrm{R}^{3}=\mathrm{H}$

b $\mathrm{R}^{1}=\mathrm{H}, \mathrm{R}^{2}=\mathrm{H}, \mathrm{R}^{3}=\mathrm{Br}$

c $R^{1}=F, R^{2}=H, R^{3}=H$

d $R^{1}=H, R^{2}=F, R^{3}=H$

e $R^{1}=H, R^{2}=H, R^{3}=F$

\section{Acknowledgements}

We greatly acknowledge the Higher Education Commission of Pakistan, Islamabad, as well as University of the Punjab, Lahore and Loughborough University for financial support. We thank $\mathrm{Mr}$ John C. Kershaw for running mass spectra and Mr J. Alastair Daley for elemental analysis.

\section{Experimental}

\section{General}

Bromoacetophenones, chloroacetophenones and fluoroacetophenones were obtained commercially from Lancaster Research Chemicals and 2-formylphenoxyactic acid from the Aldrich Chemical Company. ${ }^{1} \mathrm{H}-\mathrm{NMR}$ and ${ }^{13} \mathrm{C}-\mathrm{NMR}$ spectra were recorded on a Bruker DPX-400 instrument at 400 and $100 \mathrm{MHz}$, respectively. Chemical shifts are reported in ppm referenced to the residual solvent signal. IR spectra were recorded on a Perkin Elmer Paragon 1000 spectrometer. Mass spectra were recorded on a Jeol SX-102 instrument using FAB ionization. Melting points were recorded on a Stuart Scientific-SMP3 apparatus and are uncorrected.

Synthesis of 2-amino-4-(2'-bromophenyl)-thiazole (1a)

Method A: The title compound was prepared by addition of resublimed iodine ( $2.54 \mathrm{~g}, 0.01 \mathrm{~mol}$ ) to 2'-bromoacetophenone (1.99 g, $0.01 \mathrm{~mol}$ ) and thiourea (1.52 g, $0.02 \mathrm{~mol})$, followed by heating of the mixture overnight in an oil bath at $100{ }^{\circ} \mathrm{C}$. After cooling, the reaction mixture was triturated with diethyl ether (ca. $50 \mathrm{~mL}$ ) to remove any unreacted iodine and bromoacetophenone. The solid residue was put in cold distilled water $(200 \mathrm{~mL}$ ) and treated with $25 \%$ aqueous ammonium hydroxide (to $\mathrm{pH}$ 9-10). The precipitated thiazole was collected and purified by crystallization from hot ethanol. The yield was 85\% and m.p. $123{ }^{\circ} \mathrm{C}$; FABMS: $\mathrm{m} / \mathrm{z} 255\left(\mathrm{MH}^{+}\right)$, in agreement with the molecular formula 
$\mathrm{C}_{9} \mathrm{H}_{7} \mathrm{BrN}_{2} \mathrm{~S}$; IR; IR $\left(v_{\max }, \mathrm{KBr}, \mathrm{cm}^{-1}\right)$ : 3320 (d of $\left.\mathrm{NH}_{2}\right)$; 1510, 1460, 1045 (characteristic of the thiazole nucleus); ${ }^{1} \mathrm{H}-\mathrm{NMR}\left(\mathrm{MeOH}_{-} \mathrm{d}_{4}\right): \delta 6.87(1 \mathrm{H}, \mathrm{s}$, thiazole $\mathrm{H}-5), 7.34(1 \mathrm{H}, \mathrm{td}, J$ 11.6, $1.6 \mathrm{~Hz}, \mathrm{ArH}), 7.45$ $(1 \mathrm{H}$, td. $J$ 7.6,1.6 Hz, ArH), 7.58 (1H, dd, $J$ 7.2, $1.2 \mathrm{~Hz}, \mathrm{ArH}), 7.72\left(1 \mathrm{H}, \mathrm{dd}, J\right.$ 8.0,1.2 Hz, ArH); ${ }^{13} \mathrm{C}-$ NMR (MeOH-d $\left.{ }_{4}\right): \delta 171.2,144.6,134.9,133.1,131.7,130.0,128.8,123.4,107.7$; Anal. Calcd. for $\mathrm{C}_{9} \mathrm{H}_{7} \mathrm{BrN}_{2} \mathrm{~S}$ : C, 42.37; H, 2.77; N, 10.98; Found: C, 42.39; H, 2.76; N, 10.96.

Method B: 2-Amino-4-(2'-bromophenyl)-thiazole was also prepared following the reported procedure [14]. The spectroscopic data of the compound 1a thus prepared were identical to those given above.

The following compounds were prepared by Method A, as described above:

2-amino-4-(4'-bromophenyl)thiazole (1b)

Yield: 80\%; m.p. $178{ }^{\circ} \mathrm{C}$; FABMS: $\mathrm{m} / \mathrm{z} 255\left(\mathrm{MH}^{+}\right)$, in agreement with the molecular formula $\mathrm{C}_{9} \mathrm{H}_{7} \mathrm{BrN}_{2} \mathrm{~S}$; IR $\left(v_{\max }, \mathrm{KBr}, \mathrm{cm}^{-1}\right)$ : $3320\left(\mathrm{~d}\right.$ of $\left.\mathrm{NH}_{2}\right)$; 1515, 1455, 1050 (characteristic of the thiazole nucleus); ${ }^{1} \mathrm{H}-\mathrm{NMR}\left(\mathrm{DMSO}_{\mathrm{d}}\right.$ ): $\delta$ 8.7-7.8 (2H, bs, $\mathrm{NH}_{2}$ ), 7.67 (4H, s, ArH), 7.07 (1H, s, thiazole H-5); ${ }^{13} \mathrm{C}-\mathrm{NMR}$ (DMSO-d $\mathrm{d}_{6}$ ): $\delta$ 169.7, 140.7, 131.8, 129.5, 127.8, 122.0, 103.5; Anal. Calcd. for $\mathrm{C}_{9} \mathrm{H}_{7} \mathrm{BrN}_{2} \mathrm{~S}$ : C, 42.37; H, 2.77; N, 10.98; Found: C, 42.35; H, 2.75; N, 10.97.

\section{2-amino-4-(2'-fluorophenyl)thiazole (1c)}

Yield: 97\%; FABMS: $\mathrm{m} / \mathrm{z} 195\left(\mathrm{MH}^{+}\right)$, in agreement with the molecular formula $\mathrm{C}_{9} \mathrm{H}_{7} \mathrm{FN}_{2} \mathrm{~S}$; IR $\left(v_{\max }, \mathrm{KBr}, \mathrm{cm}^{-1}\right): 3320$ (d of $\mathrm{NH}_{2}$ ); 1510, 1455, 1050 (characteristic of the thiazole nucleus); ${ }^{1} \mathrm{H}-\mathrm{NMR}$ (MeOH-d $\left.{ }_{4}\right): \delta 6.93(1 \mathrm{H}, \mathrm{s}$, thiazole $\mathrm{H}-5), 7.13-7.36(3 \mathrm{H}, \mathrm{m}, \mathrm{ArH}), 7.93$ (1H, td, J 7.9, $\left.1.7 \mathrm{~Hz}, \mathrm{ArH}\right)$; ${ }^{13} \mathrm{C}-\mathrm{NMR}\left(\mathrm{MeOH}-\mathrm{d}_{4}\right): \delta 170.7,145.1,133.5,133.4,132.4,131.4,130.8,128.2$ and 107.9. Anal. Calcd. for $\mathrm{C}_{9} \mathrm{H}_{7} \mathrm{FN}_{2} \mathrm{~S}$ : C, 55.66; H, 3.63; N, 14.42; Found: C, 55.62; H, 3.59; N, 14.41.

\section{2-amino-4-(3’-fluorophenyl)thiazole (1d)}

Yield: 86\%; FABMS: $m / z 194\left(\mathrm{MH}^{+}\right)$, in agreement with the molecular formula $\mathrm{C}_{9} \mathrm{H}_{7} \mathrm{FN}_{2} \mathrm{~S}$; IR $\left(v_{\max }, \mathrm{KBr}, \mathrm{cm}^{-1}\right): 3320$ (d of $\mathrm{NH}_{2}$ ); 1510, 1460, 1045 (characteristic of the thiazole nucleus); ${ }^{1} \mathrm{H}-\mathrm{NMR}$ $\left(\mathrm{MeOH}-\mathrm{d}_{4}\right): \delta 7.01$ (s, thiazole $\left.\mathrm{H}-5\right), 7.09-7.56$ (4H, m, ArH); ${ }^{13} \mathrm{C}-\mathrm{NMR}\left(\mathrm{MeOH}-\mathrm{d}_{4}\right): \delta 171.8,165.7$, 163.3, 135.9, 131.7, 122.8, 116.2, 113.8 and 104.5. Anal. Calcd. for $\mathrm{C}_{9} \mathrm{H}_{7} \mathrm{FN}_{2} \mathrm{~S}$ : C,55.66; H, 3.63, N, 14.42; Found: C, 55.60; H, 3.58, N, 14.39 .

\section{2-amino-4-(4'-fluorophenyl)thiazole (1e)}

Yield: 87\%; FABMS: $\mathrm{m} / \mathrm{z} 195\left(\mathrm{MH}^{+}\right)$, in agreement with the molecular formula $\mathrm{C}_{9} \mathrm{H}_{7} \mathrm{FN}_{2} \mathrm{~S}$; IR

$\left(v_{\max }, \mathrm{KBr}, \mathrm{cm}^{-1}\right.$ ): 3320 (d of $\mathrm{NH}_{2}$ ); 1510, 1460,1045 (characteristic of the thiazole nucleus); ${ }^{1} \mathrm{H}-\mathrm{NMR}$ $\left(\mathrm{Py}_{-} \mathrm{d}_{5}\right): \delta 7.31$ (2H, brs, $\left.\mathrm{NH}_{2}\right), 8.10(1 \mathrm{H}, \mathrm{s}$, thiazole $\mathrm{H}-5), 8.18-9.20(4 \mathrm{H}, \mathrm{m}, \mathrm{ArH}) ;{ }^{13} \mathrm{C}\left(\mathrm{Py}-\mathrm{d}_{5}\right): \delta$ 171.4, 165.2, 162.7, 133.9, 129.9, 129.8, 117.3, 117.1 and 103.5; Anal. Calcd. for $\mathrm{C}_{9} \mathrm{H}_{7} \mathrm{~N}_{2} \mathrm{FS}$ : C, 55.66; H, 3.63; N, 14.42; Found: C, 55.65; H, 3.61; N, 14.40. 
Preparation of (2-\{[4-(2-bromophenyl)thiazol-2-ylimino]methyl $\}$ phenoxy)acetic acid (2a):

2-Formylphenoxyacetic acid (4.0 mmol, $0.72 \mathrm{~g}$ ) was added to 2-amino-4-(2'-bromophenyl)thiazole (4.0 mmol, $1.02 \mathrm{~g})$ in absolute EtOH $(20 \mathrm{~mL})$ in addition to molecular sieves ( $4 \AA$, $c a .5 \mathrm{~g})$ and $\mathrm{Na}_{2} \mathrm{SO}_{4}$ (anhydr. ca. $5 \mathrm{~g}$ ) and refluxed (oil bath at $90{ }^{\circ} \mathrm{C}$ ) for 3 days under $\mathrm{N}_{2}$ (g). After filtration, evaporation and recrystallisation from $\mathrm{EtOH}$ the yield of the title Schiff base was found to be $60 \%$; m.p. $180{ }^{\circ} \mathrm{C}$; HRMS (FAB, $\mathrm{MH}^{+}$) calcd. for $\mathrm{C}_{18} \mathrm{H}_{13} \mathrm{~N}_{2} \mathrm{O}_{3} \mathrm{BrS}$ : 416.9909, found: 416.9904; IR ( $v_{\max }$, $\mathrm{KBr}, \mathrm{cm}^{-1}$ ): 3030, 1635, 1550, $1240 \mathrm{~cm}^{-1}$; ${ }^{1} \mathrm{H}-\mathrm{NMR},\left(\mathrm{MeOH}-\mathrm{d}_{4}\right): \delta 5.77$ (2H, s, $\left.\mathrm{CH}_{2}\right), 6.91(1 \mathrm{H}, \mathrm{d}, J$ $9.2 \mathrm{~Hz}, \mathrm{ArH}), 7.46-7.73(7 \mathrm{H}, \mathrm{m}, \mathrm{ArH}), 7.92(1 \mathrm{H}, \mathrm{s} \mathrm{CH}=\mathrm{N}) ;{ }^{13} \mathrm{C}-\mathrm{NMR}\left(\mathrm{MeOH}-\mathrm{d}_{4}\right): \delta$ 67.1, 113.4, 122.1, 122.3, 128.4, 129.5, 130.0, 130.8, 131.1, 130.3, 133.2, 133.8, 136.4, 144.9, 156.3, 1661.5,168.9, 172.0; Anal. Calcd. for $\mathrm{C}_{18} \mathrm{H}_{13} \mathrm{BrN}_{2} \mathrm{O}_{3} \mathrm{~S}$ : C, 51.81; H, 3.14; N, 6.71. Found: C, 51.79, H, 3.12; N, 6.69;

Preparation of (2-\{[4-(4-bromophenyl)-thiazole-2-ylimino]methyl\}phenoxy)acetic acid (2b)

2-Formylphenoxyacetic acid (4.0 mmol, $0.72 \mathrm{~g}$ ) was added to a solution of 2-amino-4-(4'-

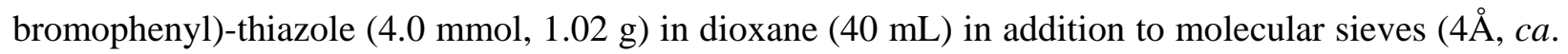
$5 \mathrm{~g}$ ) and $\mathrm{Na}_{2} \mathrm{SO}_{4}$ (anhydr. ca. $5 \mathrm{~g}$ ) and refluxed above $100{ }^{\circ} \mathrm{C}$ for 2 days under $\mathrm{N}_{2}$ (g). The product was purified by crystallization from EtOH and the yield of the Schiff base was found to be 67\%; m.p. 185$187^{\circ} \mathrm{C}$; HRMS (FAB, $\mathrm{MH}^{+}$) calcd. for $\mathrm{C}_{18} \mathrm{H}_{13} \mathrm{BrO}_{3} \mathrm{~N}_{2} \mathrm{~S}$ : 416.9909, found: 416.9904; IR (v $v_{\max }, \mathrm{KBr}$, $\mathrm{cm}^{-1}$ ): 3030, 1650, 1550, 1240. Anal. Calcd. for $\mathrm{C}_{18} \mathrm{H}_{13} \mathrm{BrO}_{3} \mathrm{~N}_{2} \mathrm{~S}$ : C, 51.81; H, 3.14; N, 6.71. Found: C, 51.80, H, 3.10; N, 6.70; ${ }^{1} \mathrm{H}-\mathrm{NMR},\left(\mathrm{MeOH}_{-} \mathrm{d}_{4}\right): \delta 6.17\left(2 \mathrm{H}, \mathrm{s}, \mathrm{CH}_{2}\right), 6.77$ (1H, d, J 8.0 Hz, ArH) 7.10$7.63(7 \mathrm{H}, \mathrm{m}, \mathrm{ArH}), 7.99(1 \mathrm{H}, \mathrm{s}, \mathrm{CH}=\mathrm{N}) ;{ }^{13} \mathrm{C}-\mathrm{NMR}\left(\mathrm{MeOH}-\mathrm{d}_{4}\right): \delta$ 65.9,113.4, 122.8, 122.9, 123.9, 124.4, 129.4, 129.8, 130.7, 131.0, 131.1, 131.2, 132.8, 132.9, 140.1, 156.3, 160.9, 172.0

Preparation of (2-\{[4-(2'-fluorophenyl)-thiazole-2-ylimino]methyl $\}$ phenoxy)acetic acid (2c)

2-Formylphenoxyacetic acid (1.0 mmol, $0.18 \mathrm{~g})$ was added to a solution of 2-amino-4-(2'fluorophenyl)-thiazole $(1.0 \mathrm{mmol}, 0.19 \mathrm{~g})$ in absolute EtOH $(10 \mathrm{~mL})$ in addition to $10 \% \mathrm{mmol}$ of $\mathrm{Yb}(\mathrm{OTf})_{3}$ as Lewis catalyst and refluxed for 10 hours under $\mathrm{N}_{2}$ (g). The reaction mixture was filtered through a column of silica gel, charcoal and Celite ${ }^{\circledR}$ to remove the catalyst. After evaporation of the ethanol, the product was purified by recrystallisation from $\mathrm{CHCl}_{3} / \mathrm{MeOH}$ (a few drops) to give the Schiff base in $70 \%$ yield; m.p. $167{ }^{\circ} \mathrm{C}$; HRMS (FAB, $\mathrm{MH}^{+}$) calcd. for $\mathrm{C}_{18} \mathrm{H}_{13} \mathrm{FN}_{2} \mathrm{O}_{3} \mathrm{~S}$ : 357.0709, found: 357.0712; IR ( $\left.v_{\max }, \mathrm{KBr}, \mathrm{cm}^{-1}\right)$ : 3030, 1640, 1550, 1240; ${ }^{1} \mathrm{H}-\mathrm{NMR},\left(\mathrm{MeOH}-\mathrm{d}_{4}\right): \delta 5.64(2 \mathrm{H}, \mathrm{s}$, $\left.\mathrm{CH}_{2}\right)$, 6.79-7.68 (8H, m, ArH), $7.99(1 \mathrm{H}, \mathrm{s}, \mathrm{CH}=\mathrm{N}) ;{ }^{13} \mathrm{C}-\mathrm{NMR}\left(\mathrm{MeOH}-\mathrm{d}_{4}\right): \delta 66.3,116.8,122.2$, 122.5, 125.1, 126.3, 128.1, 128.4, 129.3, 130.3, 130.9, 132.9, 138.2, 140.1, 157.2, 160.1, 169.5, 172.8; Anal. calcd. for $\mathrm{C}_{18} \mathrm{H}_{13} \mathrm{FN}_{2} \mathrm{O}_{3} \mathrm{~S}$ : C, 51.81; H, 3.14; N, 6.71. Found: C, 51.60, H, 3.08; N, 6.64;

Preparation of (2-\{[4-(3’-fluorophenyl)thiazole-2-ylimino]methyl $\}$ phenoxy)acetic acid (2d)

Compound 2d was synthesized by the method described above. The product was purified by crystallization from chloroform and the yield of the title Schiff base was 70\%; m.p. $178{ }^{\circ} \mathrm{C}$ (decomp.); HRMS (FAB, $\mathrm{MH}^{+}$) calcd. for $\mathrm{C}_{18} \mathrm{H}_{13} \mathrm{FN}_{2} \mathrm{O}_{3} \mathrm{~S}$ : 357.0709; found: 357.0712; IR ( $v_{\max }, \mathrm{KBr}, \mathrm{cm}^{-1}$ ): 3030, 1635, 1550, 1240; ${ }^{1} \mathrm{H}-\mathrm{NMR}\left({\left.\mathrm{MeOH}-\mathrm{d}_{4}\right): \delta} 6.15\right.$ (2H, s, $\mathrm{CH}_{2}$ ), 6.93-7.37 (8H, m, ArH), 7.91 (1H, s, 
$\mathrm{CH}=\mathrm{N}) ;{ }^{13} \mathrm{C}-\mathrm{NMR}\left({\left.\mathrm{MeOH}-\mathrm{d}_{4}\right): \delta}_{6} 66.3,113.4,116.4,122.3,124.6,124.9,125.3,129.4,130.4,131.2\right.$, 131.3, 131.5, 131.6, 138.9, 140.9, 157.9, 162.4, 169.7; Anal. Calcd. for $\mathrm{C}_{18} \mathrm{H}_{13} \mathrm{FN}_{2} \mathrm{O}_{3} \mathrm{~S}$ : C, 51.81; $\mathrm{H}$, 3.14; N, 6.71. Found: C, 51.64, H, 3.10; N, 6.56.

Preparation of (2-\{[4-(4'-fluorophenyl)-thiazole-2-ylimino]methyl\}phenoxy)acetic acid (2e)

Compound 2e was synthesized by the method described above. The product was purified by crystallization from chloroform and the yield of the Schiff base was 70\%; m.p. 158-160 ${ }^{\circ} \mathrm{C}$; HRMS (FAB, $\mathrm{MH}^{+}$) calcd. for $\mathrm{C}_{18} \mathrm{H}_{13} \mathrm{FN}_{2} \mathrm{O}_{3} \mathrm{~S}$ : 357.0709, found: 357.0713; IR (v $v_{\max }, \mathrm{KBr}, \mathrm{cm}^{-1}$ ): 3030, 1630, 1550, 1240; ${ }^{1} \mathrm{H}-\mathrm{NMR}\left(\mathrm{MeOH}-\mathrm{d}_{4}\right): \delta 6.09\left(2 \mathrm{H}, \mathrm{s}, \mathrm{CH}_{2}\right), 6.90-7.48(8 \mathrm{H}, \mathrm{m}, \mathrm{ArH}), 7.91(1 \mathrm{H}, \mathrm{s}, \mathrm{CH}=\mathrm{N})$;

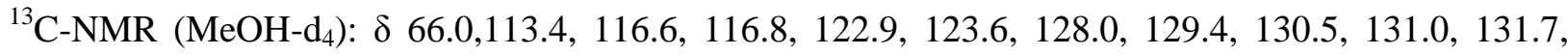
131.8, 138.9, 140.6, 156.4,163.2, 165.7, 171.6; Anal. Calcd. for $\mathrm{C}_{18} \mathrm{H}_{13} \mathrm{FN}_{2} \mathrm{O}_{3} \mathrm{~S}$ : C, 51.81; H, 3.14; N, 6.71. Found: C, 51.59, H, 3.40; N, 6.49;

\section{References}

1. Karia, F. D.; Parsania, P.H. Synthesis, biological and thermal properties of Schiff bases of bisphenol-C. Asian J. Chem. 1999, 11, 991-995.

2. More, P. G.; Bhalvankar, R. B.; Pattar, S. C. Synthesis and biological activities of Schiff bases of aminothiazoles. J. Indian Chem. Soc. 2001, 78, 474-475.

3. El-Masry, A. H.; Fahmy, H. H.; Abdelwahed, S. H. A. Synthesis and antimicrobial activity of some new benzimidazole derivatives. Molecules 2000, 5, 1429-1438.

4. Baseer, M. A.; Jadhav, V. D.; Phule, R. M.; Archana, Y. V.; Vibhute, Y. B. Synthesis and antimicrobial activity of some new Schiff bases, Orient. J. Chem. 2000, 16, 553-556.

5. Pandeya, S. N.; Sriram, D.; Nath, G.; De Clercq, E. Synthesis and antimicrobial activity of Schiff and Mannich bases of isatin and its derivatives with pyrimidine, IL Farmaco 1999, 54, 624-628.

6. Singh, W. M.; Dash, B. C. Synthesis of some new Schiff bases containing thiazole and oxazole nuclei and their fungicidal activity, Pesticides 1988, 22, 33-37.

7. Hodnett, E. M. and Dunn, W. J. Structure-antitumour activity correlation of some Schiff bases, $J$. Med. Chem. 1970, 13, 768-770.

8. Desai, S. B.; Desai, P. B.; Desai, K.R. Synthesis of some Schiff bases, thiazolidones, and azetidinones derived from 2,6-diaminobenzo[1,2-d:4,5-d']bisthiazole and their anticancer activities, Heterocycl. Commun. 2001, 7, 83-90.

9. Pathak, P.; Jolly, V. S.; Sharma, K.P. Synthesis and biological activities of some new substituted arylazo Schiff bases, Oriental. J. Chem. 2000, 16, 161-162.

10. Samadhiya, S.; Halve, A. Synthetic utility of Schiff bases as potential herbicidal agents, Orient. J. Chem. 2001, 17, 119-122.

11. Aydogan, F.; Öcal, N.; Turgut, Z.; Yolacan, C. Transformations of aldimines derived from pyrrole-2-carboxaldehyde. Synthesis of thiazolidino-fused compounds, Bull. Korean Chem. Soc. 2001, 22, 476-480.

12. Taggi, A. E.; Hafez, A. M.; Wack, H.; Young, B.; Ferraris, D.; Lectka, T. The development of the first catalysed reaction of ketenes and imines: catalytic asymmetric synthesis of $\beta$-lactams, $J$. Am. Chem. Soc. 2002, 124, 6626-6635. 
13. King, L.C; Hlavacek, R.J. Reaction of ketones with iodine and thiourea, J. Am. Chem. Soc. 1950, 72, 3722.

14. Zav’Yalov, S.I.; Dorofeeva, O.V.; Rumyantseva, E.E.; Kulikova, L.B.; Ezhova, G.I.; Kravchenko N.E.; Zavozin, A.G. Synthesis of 2-aminothiazole derivatives, Pharm. Chem. J. 2001, 35, 96-98.

Sample availability: Contact the authors

(C) 2006 by MDPI (http://www.mdpi.org). Reproduction is permitted for noncommercial purposes. 